This item was submitted to Loughborough's Research Repository by the author.

Items in Figshare are protected by copyright, with all rights reserved, unless otherwise indicated.

\title{
Tailoring surface hydrophilicity of porous electrospun nanofibers to enhance capillary and push-pull effects for moisture wicking
}

PLEASE CITE THE PUBLISHED VERSION

http://dx.doi.org/10.1021/am503417w

\section{PUBLISHER}

(C) American Chemical Society

\section{VERSION}

AM (Accepted Manuscript)

\section{PUBLISHER STATEMENT}

This work is made available according to the conditions of the Creative Commons Attribution-NonCommercialNoDerivatives 4.0 International (CC BY-NC-ND 4.0) licence. Full details of this licence are available at: https://creativecommons.org/licenses/by-nc-nd/4.0/

\section{LICENCE}

CC BY-NC-ND 4.0

\section{REPOSITORY RECORD}

Dong, Yuliang, Junhua Kong, Si Lei Phua, Chenyang Zhao, Noreen L. Thomas, and Xuehong Lu. 2014. "Tailoring Surface Hydrophilicity of Porous Electrospun Nanofibers to Enhance Capillary and Push-pull Effects for Moisture Wicking". figshare. https://hdl.handle.net/2134/16070. 


\section{Tailoring surface hydrophilicity of porous}

\section{electrospun nanofibers to enhance capillary and}

\section{push-pull effects for moisture wicking}

Yuliang Dong ${ }^{1,2,3, \#}$, Junhua Kong ${ }^{1, \#}$, Si Lei Phua ${ }^{1}$, Chenyang Zhao ${ }^{1}$, Noreen L. Thomas ${ }^{3}$, Xuehong $L u^{1,2, *}$

1 School of Materials Science and Engineering, Nanyang Technological University, 50 Nanyang Avenue, Singapore 639798

2 Institute for Sport Research, Nanyang Technological University, 50 Nanyang Avenue, Singapore 639798

3 Department of materials, Loughborough University, Loughborough, Leicestershire, LE11 3TU, United Kingdom

KEYWORDS

moisture transport, porous nanofibers, electrospinning, capillary motion, push-pull, wetting

\section{ABSTRACT}

In this article, liquid moisture transport behaviors of dual-layer electrospun nanofibrous mats are reported for the first time. The dual-layer mats consist of a thick layer of hydrophilic 
polyacrylonitrile (PAN) nanofibers with a thin layer of hydrophobic polystyrene (PS) nanofibers with and without interpenetrating nanopores, respectively. The mats are coated with polydopamine (PDOPA) to different extents to tailor the water wettability of the PS layer. It is found that with a large quantity of nanochannels, the porous PS nanofibers exhibit a stronger capillary effect than the solid PS nanofibers. The capillary motion in the porous PS nanofibers can be further enhanced by slight surface modification with PDOPA while retaining the large hydrophobicity difference between the two layers, inducing a strong push-pull effect to transport water from the PS to the PAN layer.

\section{Introduction}

In the fields of sportswear, activewear and workwear, moisture transport behavior of textile is an important issue because it greatly influences the thermo-physiological comfort of the human body. For example, in order to maintain the thermal balance of people during sports activities in hot outdoor environments, heat needs to be transported out quickly by perspiration. The inefficient transport of sweat through sports apparels causes discomfort, and the excessive heat accumulated may cause heat stress and affect sports performance. In order to achieve good moisture transport properties, single-layer textiles consisting of synthetic hydrophobic microfibers that have low water absorption capability, such as polyester and polypropylene (PP), are widely used in commercial sportswear owing to their good moisture release capability. ${ }^{1}$ To further enhance the wicking ability of the textiles, hydrophilization of hydrophobic fibers via surface modification is an effective strategy. For instance, Masuduz et al. ${ }^{2}$ modified the surface 
of polyester textile by applying a hydrophilic surface finishing agent that contains nanocrystalline cellulose, leading to improved water wettability. The enhancement of the water wettability of PP fibers by surface adsorption of different soybean proteins has also been reported. ${ }^{3}$

Compared with single-layer textiles, dual-layer textiles have great potential to achieve better moisture transport properties because the components and structures of the two layers can be tailored independently. Dual-layer moisture-wicking textiles are usually composed of a hydrophilic outer layer and a hydrophobic inner layer. A typical example has cotton as the outer layer and PP as the inner layer. ${ }^{4}$ In such textiles, the hydrophobic inner layer ensures low water absorption and transmission of sweat via capillary motion, while the hydrophilic outer layer draws the sweat out from the inner layer and facilitates evaporation to the surrounding environment. The capillary motion of the inner layer is induced by the intermolecular interactions between the fiber surface and sweat, and can be controlled by tailoring the size of interpenetrating pores and water wettability of the textiles. The smaller the pore size and the better the wettability, the stronger the capillary effect. Overall, the moisture transport property of the dual-layer textiles strongly depends on the hydrophobicity difference between the two layers, which induces the push-pull force, as well as the morphology and structure of the inner layer, which dominates the capillary motion.

Electrospinning has been established as a simple and scalable method for fabrication of nonwoven mats of polymer nanofibers. ${ }^{5-7}$ The diameter of the nanofibers and the thickness of the 
nanofibrous mats can be easily controlled by adjusting various processing parameters. ${ }^{8}$ The free standing nature of the electrospun nanofibrous mats gives them the potential to be directly used as functional textiles. ${ }^{9-10}$ Some electrospun webs have also been assessed as barriers to liquid penetration for their potential use in protective clothing. ${ }^{11}$ In terms of transport properties, previous research demonstrated that the electrospun mats with micron-sized inter-fiber spaces exhibited minimal impedance to moisture vapor permeation required for evaporative cooling, ${ }^{12}$ and their water vapor permeability was comparable to that of normal woven sportwear and workwear. ${ }^{13}$ However, so far there is no report on liquid moisture transport behaviors of electrospun nonwoven mats. Moreover, nanometer-sized pores can be created in electrospun nanofibers by adjusting solvent composition or spinning environment humidity. ${ }^{14-16}$ Also surface hydrophilicity of nanofibers can be tailored via various surface modification processes, ${ }^{17-18}$ although these opportunities to enhance the capillary effect have not been explored.

In this study, for the first time polyacrylonitrile (PAN), polystyrene (PS) and PAN-PS duallayer electrospun nanofibrous mats have been fabricated to investigate liquid moisture transport behaviors through the electrospun mats. PAN is a commonly used textile material and PAN nanofibrous mats are chosen as the outer hydrophilic layer in the dual-layer system. PS is a common hydrophobic polymer that can be electrospun more easily than polyesters and PP, and hence PS nanofibrous mats are chosen as the inner phydrophobic layer. Both solid and porous electrospun PS nanofibers were fabricated by manipulating solvent composition and their surface were coated with polydopamine (PDOPA), which is a mimic of essential components of mussel adhesive proteins ${ }^{19}$ and rich in hydrophilic groups, to achieve different degrees of hydrophilicity, yielding inner layers with different capillary effects. PDOPA was used to tailor the surface 
hydrophilicity of the PS mats because a very thin PDOPA layer could be facilely deposited onto the surface of the nanofibers via in-situ polymerization ${ }^{20}$ owing to its excellent adhesive properties and also because the coating thickness could be well controlled by adjusting the polymerization conditions and time. ${ }^{21}$ Herein we report the effects of nanofiber morphology and surface hydrophilicity on the liquid moisture transport properties of the electrospun mats. The possibility of significantly enhancing the push-pull effect in dual-layer mats by tailoring the structure and morphology of the inner layer is also demonstrated.

\section{Experimental}

Materials. PAN $\left(\mathrm{M}_{\mathrm{w}}=150000\right), \mathrm{PS}\left(\mathrm{M}_{\mathrm{w}}=350000\right)$, dopamine hydrochloride (DOPA) and tris(hydroxymethyl) aminomethane (Tris) were purchased from Sigma-Aldrich Chemistry (USA). Dimethylformamide (DMF) and tetrahydrofuran (THF) were purchased from Tedia Company Inc (USA). Ethanol was supplied by Merck KGaA (Germany). All materials were used without further purification.

Preparation of PAN, PS nanofibers and PS-PAN dual-layer mats. PAN solution in DMF (8 wt\% PAN), PS solution in DMF (15 wt $\%$ PS) and PS solution in DMF/THF (DMF/THF weight ratio $=1: 1,15 \mathrm{wt} \%$ PS) were prepared by dissolving PAN or PS powder in the corresponding solvent(s) at $60{ }^{\circ} \mathrm{C}$ under continuous magnetic stirring. All solutions were electrospun into nonwoven nanofibrous mats using a conventional single-spinneret electrospinning setup (MECC nanon-01 A, Japan). The needle tip-to-plate collector distance was $15 \mathrm{~cm}$. The type of spinneret 
used was $27^{1 / 2} \mathrm{G}$ with inner diameter of $200 \mu \mathrm{m}$. The working voltage and flow rate were $10 \sim 13$ $\mathrm{kV}$ and $0.5 \mathrm{~mL} \mathrm{~h}^{-1}$, respectively. The mats were collected on aluminum foil. The spinning time for PAN/DMF, PS/DMF and PS/DMF/THF solutions were $3 \mathrm{hrs}, 25 \mathrm{~min}$ and $25 \mathrm{~min}$, respectively. PAN-PS double-layer mats were prepared via electrospinning of PS/DMF or PS/DMF/THF solution for 25 min followed by electrospinning of PAN/DMF solution for $3 \mathrm{hrs}$ on aluminum foils. All the mats were dried in vacuum at $60{ }^{\circ} \mathrm{C}$ for $24 \mathrm{hrs}$ and then went through a paper laminator (PRO $\mathrm{BiO} 320$ ) at $70^{\circ} \mathrm{C}$ and a rolling speed of $50 \mathrm{rpm}$.

Preparation of PDOPA coating. PDOPA was coated on the electrospun mats via in situ polymerization. In a typical experiment, an electrospun mat was immersed in $500 \mathrm{~mL} 1.21$ $\mathrm{mg} / \mathrm{mL}$ Tris buffer aqueous solution, and $150 \mathrm{mg}$ DOPA $(0.3 \mathrm{mg} / \mathrm{mL})$ was then added into the solution. The polymerization was conducted at room temperature for 15 and $90 \mathrm{~min}$, respectively, under continuous magnetic stirring. All PDOPA-coated mats were rinsed with deionized (DI) water for three times and dried in vacuum at $60{ }^{\circ} \mathrm{C}$ for $24 \mathrm{hrs}$. Solution-casted solid PAN and PS films were also coated with PDOPA following the same procedure. The films were casted from the PAN/DMF and PS/DMF/THF solutions and dried in a vacuum oven at 80 ${ }^{\circ} \mathrm{C}$ for $24 \mathrm{hrs}$.

Characterization. Morphologies of the mats were observed using a field-emission scanning electron microscope (FESEM, JEOL 7600F). FESEM images were obtained under an accelerating voltage of $5 \mathrm{kV}$, and a working distance of $8 \mathrm{~mm}$. All samples were coated with a thin layer of gold before SEM observation. In order to test water absorption by the nanofibrous 
mats, the rectangle-shaped mats with area of $20 \mathrm{~cm}^{2}$ were immersed in DI water for $48 \mathrm{hrs}$. Their weight was measured before and after immersion in the water. Water absorption is defined as the weight ratio of the absorbed water to the dry sample. Contact angles of the films and apparent contact angles of the nanofibrous mats were measured using a contact angle tester (Analytical Technologies, FTA32) via drop shape analysis. Moisture transport behaviors of the mats were characterized using a Moisture Management Tester (MMT, SDLATLAS). MMT is a useful instrument to measure the dynamic liquid transport properties of textiles. ${ }^{22}$ The electrical resistance of dry textiles is usually very large when placed in a closed circuit. When a piece of fabric is wet, the resistance is reduced and the extent of the reduction is dependent on the composition of the liquid as well as the liquid content. Using MMT, the resistance of a fabric sample could be measured on both sides of the fabric. The water contents of the two surfaces can thus be calculated by correlation with the corresponding resistances, ${ }^{22}$ and these relative water contents can be used to infer water transport behavior of the sample. The liquid used in the tests was $0.15 \mathrm{M}$ sodium chloride aqueous solution. An aqueous blue ink was used to stain the PSpNF/PAN-sNF and PS-pNF-15/PAN-sNF dual-layer mats to visually observe their liquid transport behavior. The durability of the PS-pNF/PAN-sNF dual-layer nanofibrous mat was evaluated by staining the mat with blue ink, vigorously shaking it using shaker in detergent aqueous solution $(0.15 \mathrm{~g} / 100 \mathrm{~mL})$ for 24 hours followed by shaking it in water for 1 hour, and then dried in vacuum at $60{ }^{\circ} \mathrm{C}$ for $4 \mathrm{hrs}$. The apparent contact angles of the mat before and after washing were compared.

\section{Results and Discussion}


Morphologies and surface modification of the electrospun nanofibrous mats

In this work, in order to investigate the effects of nanofiber morphology and surface hydrophilicity on water transport behavior of the electrospun nanofibrous mats, three types of nonwoven mat composed of PAN, solid and porous PS nanofibers were fabricated by conventional electrospinning and the nanofiber surfaces were coated with PDOPA to different extents.

To facilitate the discussion below, the morphological and surface features of the mats are indicated in the sample names. For example, PAN and PS solid nanofibers without PDOPA coating are denoted as PAN-sNF and PS-sNF, respectively, while PS porous nanofibers with PDOPA coating time of 90 min are denoted as PS-pNF-90. The reference samples are named in a similar way except that NF (nanofiber) is replaced by FM (film). For instance, PS-FM-15 denotes the cast PS films with PDOPA coating time of $15 \mathrm{~min}$.

As shown by the low-magnification SEM images in Figure 1, all the mats are composed of nanofibers with average diameter of less than $1 \mu \mathrm{m}$. Although the nanofiber diameters of the three types of mats are different, such moderate differences do not affect their water wettability and transport behaviors significantly (Figure S1) probably because the sizes of the inter-fiber spaces in the mats are not very different. The high-magnification FESEM images in Figure 1 confirm the solid nature of PAN-sNF and PS-sNF, for which DMF and DMF/THF mixed solvents were used, respectively, and the high porosity of PS-pNF, for which DMF was used as 
the solvent. The mechanism for the formation of porous PS nanofibers has been reported by P. Lu, et al. ${ }^{16}$

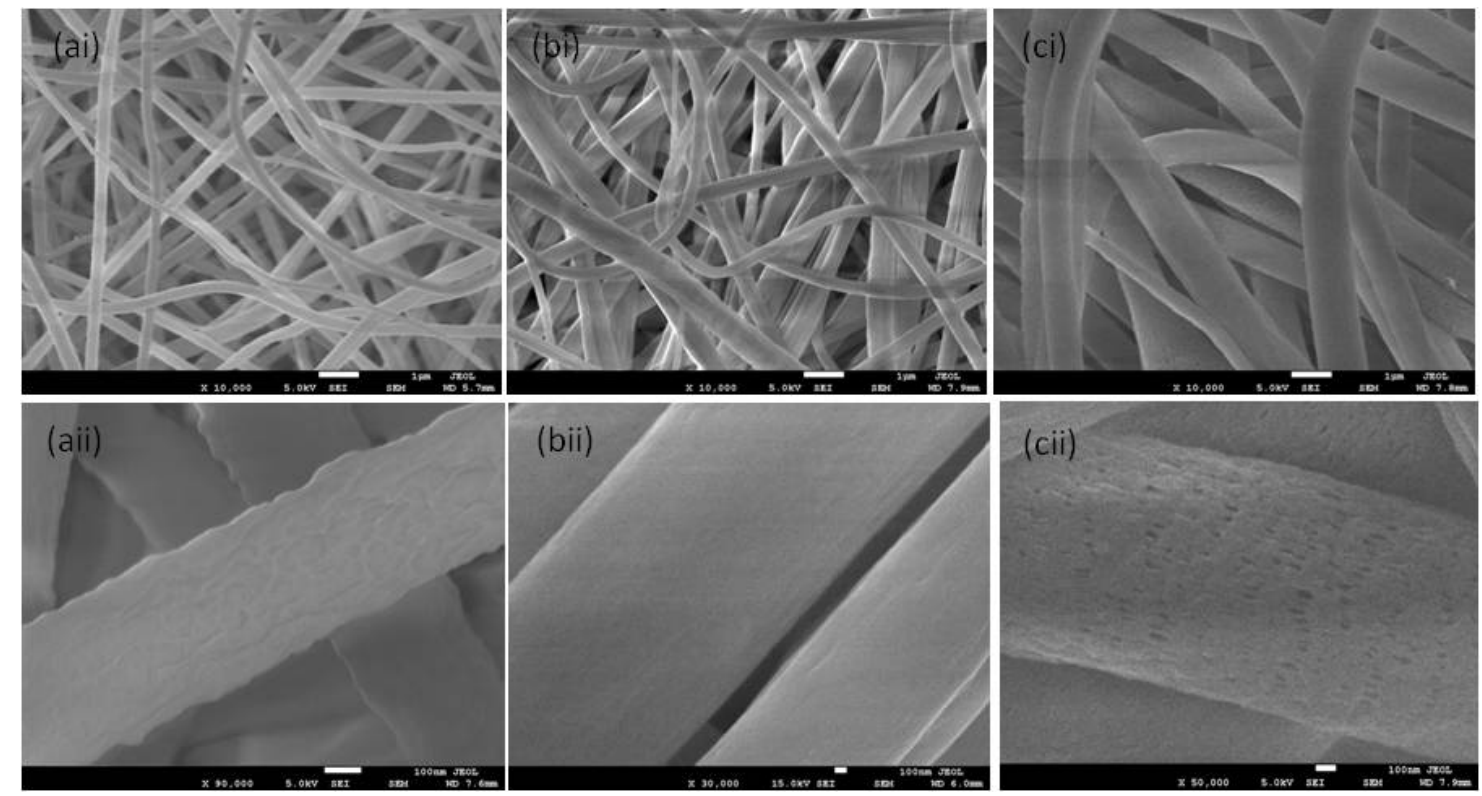

Figure 1. FESEM micrographs showing the morphologies of the as-spun (ai and aii) solid PAN (PAN-sNF), (bi and bii) solid PS (PS-sNF) and (ci and cii) porous PS nanofibers (PS-pNF).

The high porosity of PS-sNF can be seen more clearly from its cross-sectional FESEM image (Figure 2a). The pores inside the nanofibers are actually inter-penetrating nanochannels with size of less than $100 \mathrm{~nm}$. Such nanochannels give much higher specific surface area (SSA) than the solid nanofibers. More importantly, the nanometer-sized channels and their inter-penetrating nature may greatly facilitate capillary motion, benefiting water transport. 


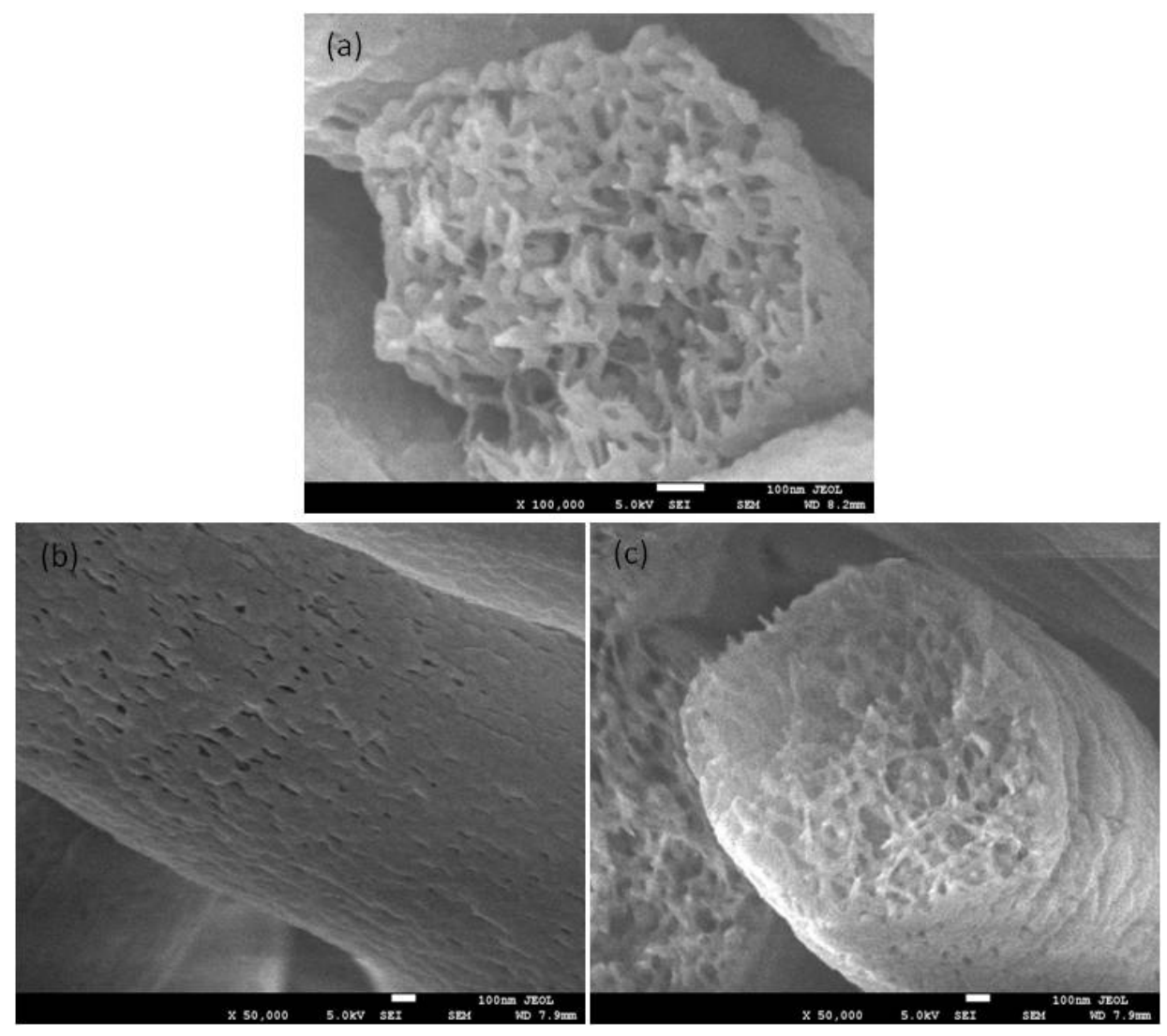

Figure 2. High-magnification FESEM micrographs showing the morphologies of (a) the as-spun porous PS nanofibers (PS-pNF), and (b) the porous PS nanofibers coated with PDOPA for 90 min (PS-pNF-90). (c) The cross-sectional FESEM image of PS-pNF-90.

As introduced above, the hydrophicility of fibers plays crucial role in determining the moisture wicking capability of textiles. In this work, PDOPA was used to tailor the surface hydrophilicity of the PS mats. As shown in Figures $2 b$ and $2 c$, the inter-penetrating pores in the porous PS nanofibers are still distinguishable after coating with PDOPA for 90 mins. For all the mats, the nanofibrous morphology was retained after PDOPA coating for 90 mins (Figure S2). 
The successful PDOPA coating was further verified by FTIR studies. To show the surface structure evolution with coating time more clearly, the spectra from solid film samples are elaborated here. As shown in Figure 3, compared with the FTIR spectrum of PS-FM, the spectra from PS-FM-15 and PS-FM-90 show characteristic peaks of PDOPA at about $3300 \mathrm{~cm}^{-1}(-\mathrm{OH}$ stretch) and $1260 \mathrm{~cm}^{-1}$ (C-O vibration of phenolic moieties), and a shoulder at $1120 \mathrm{~cm}^{-1}{ }^{23}$ With the increased coating time, these characteristic peaks become more intensive, indicating the deposition of a larger amount of PDOPA. The FTIR spectra of the nanofibrous mats are given in Supporting Information (Figure S3), which shows a similar trend to that seen from the cast films.

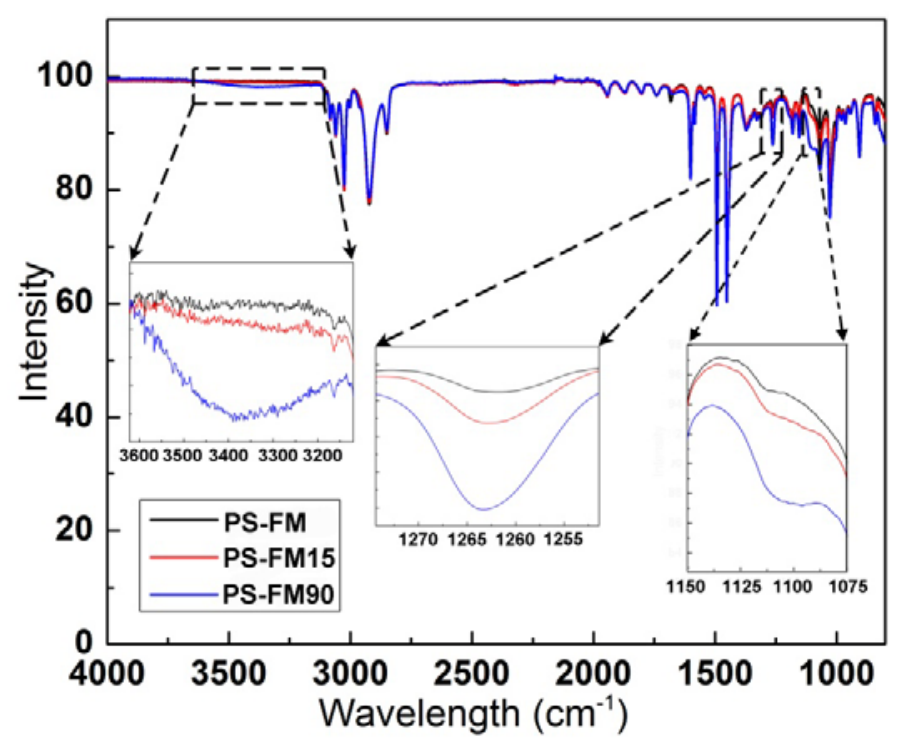

Figure 3. FTIR spectra of PS-FM, PS-FM15 and PS-FM90.

Effect of capillary effect on liquid moisture transport behavior 
To study the water transport behavior of the nanofibrous mats, understanding the interaction between the mats and water is of great importance. Thus time-dependent contact angles of the samples were measured to characterize the water wettability and spreading capability of the surfaces. For the nanofibrous mats, the measurements give apparent contact angles, which are dependent on not only the surface properties, but also the rate of water penetration into the mats. The water transport capabilities of the mats were also measured using MMT to infer water transport behavior of the sample.

Firstly, to understand the intrinsic water wettability of PAN and PS, and the effects of the PDOPA coating on their surface hydrophilicity, contact angles of the cast PAN and PS solid films before and after PDOPA coating were measured. It is clear that the PAN film exhibits smaller contact angle and hence is more hydrophilic than the PS film (Figure 4). In addition, water could spread on the PAN film in all directions uniformly, decreasing the contact angle significantly within $1 \mathrm{~min}$, whereas water cannot spread on the PS film (no significant contact angle change in $1 \mathrm{~min}$ ), showing the hydrophobic nature of PS. For the PAN film, the PDOPA coating does not cause any significant change in contact angle of the film, implying that both PAN and PDOPA surfaces are fairly hydrophilic. Upon depositing a PDOPA layer onto the PS film, the contact angle reduces slightly, which is more distinct for PS-FM-90, confirming that the PS surface has been successfully modified by PDOPA. However, even with the PDOPA coating, the contact angles of PS-FM-15 and PS-FM-90 do not decrease significantly within $1 \mathrm{~min}$, implying that the surfaces are still fairly hydrophobic due to the relatively short coating times and hence deposition of fairly small quantities of PDOPA on the PS thin films. Note that with the 
slightly reduced contact angle, the gravity force for further spreading is smaller. In addition, it is likely that with very short period of coating time, PDOPA may not fully cover the film surface.
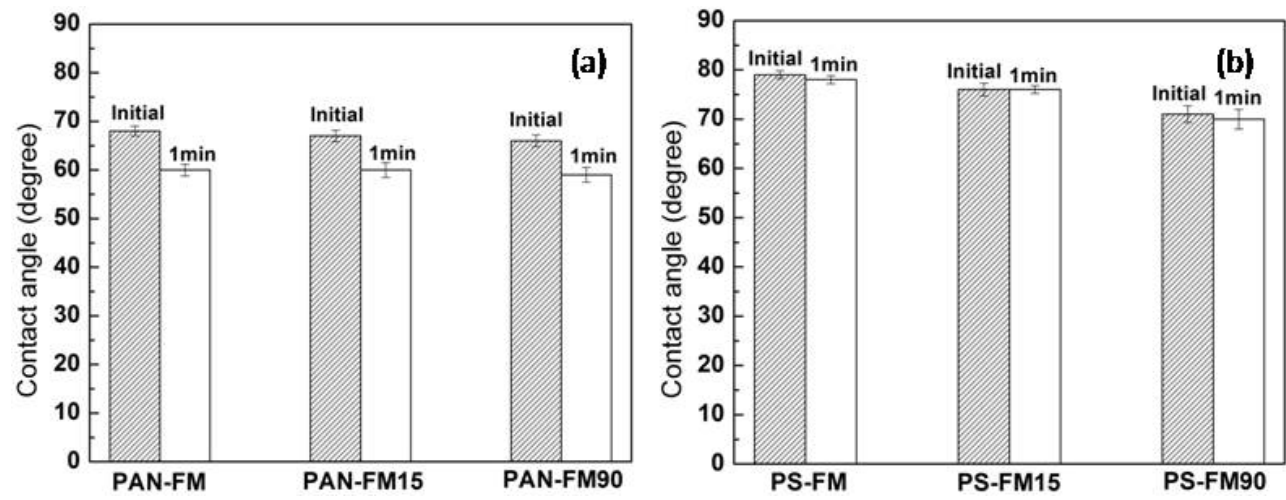

Figure 4. Contact angles of the solid films (a) PAN-FM, PAN-FM-15 and PAN-FM-90, and (b) PS-FM, PS-FM-15 and PS-FM-90.

Figures $5 \mathrm{a}$ and $5 \mathrm{c}$ show the apparent contact angles of the PAN nanofibrous mats. There is no significant difference in contact angle between PAN-sNF, PAN-sNF-15 and PAN-sNF-90. However, it is noted that the apparent contact angles of the mats are much smaller than that of corresponding cast PAN films. Although PAN has poor water sorption properties because it is highly crystalline, with the aid of surface nitrile groups, water on the top surfaces of the mats could quickly spread along/across the nanofibers, penetrate into the pores in the mats and wet the bottom surface of the mats. The apparent contact angle is therefore rapidly reduced to zero and the water drop disappears in just two seconds (Figure S4). This is further supported by the MMT results shown in Figure 5d. The solid line represents the water content of the top surface where the water drops are sprayed initially, while the dashed line represents the water content of the 
bottom surface. For PAN-sNF, the relative water contents of both surfaces increase fast initially and rapidly reach a plateau. The relative water content of the top surface is always higher than that of the lower surface, indicating that although water can pass the mat quickly, once a certain water content gradient is established, the water transport is slowed down significantly. The PDOPA coating on the PAN nanofibers has no obvious effect on the water transport property.

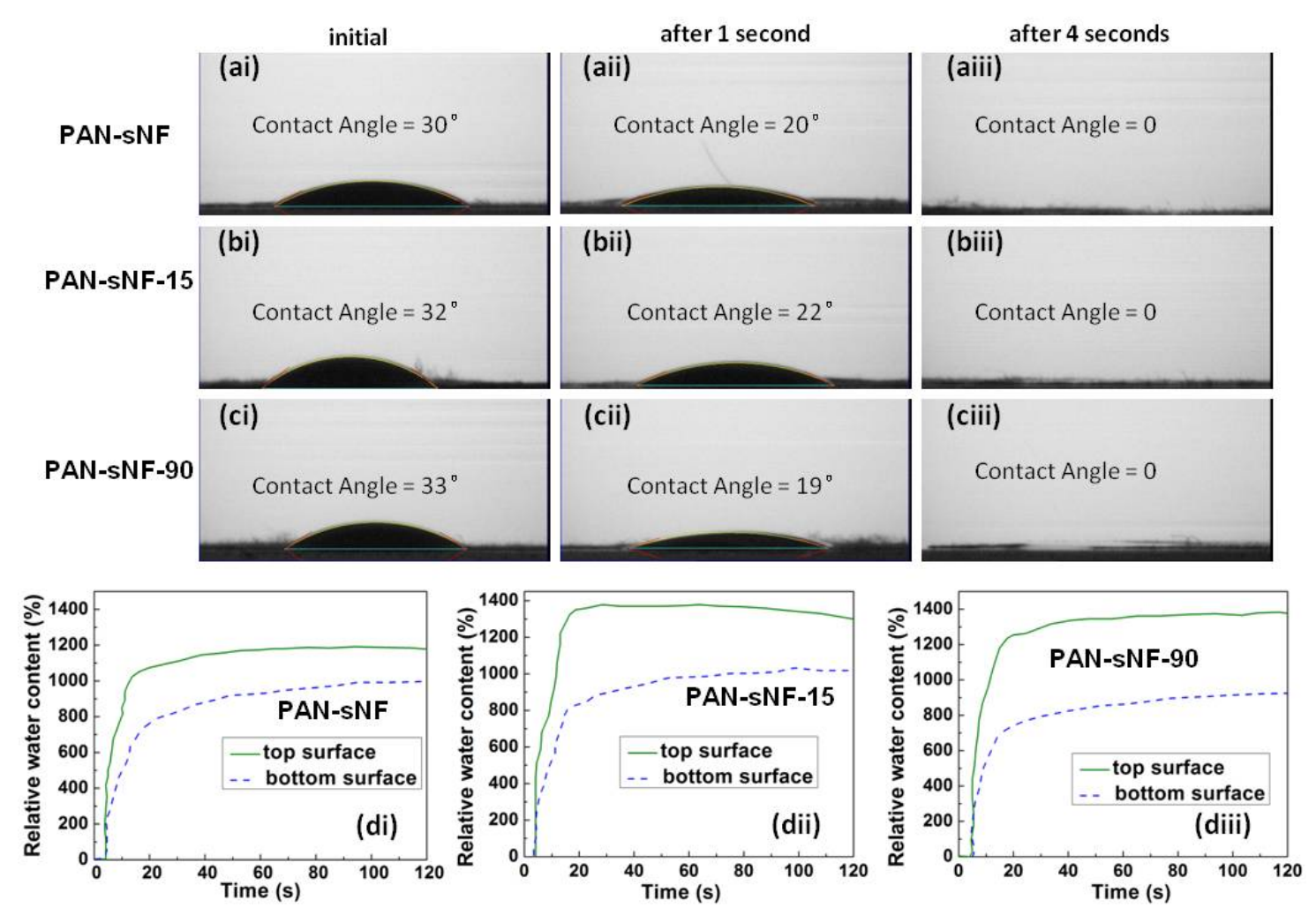

Figure 5. Apparent water contact angles of (a) PAN-sNF, (b) PAN-sNF-15 and (c) PAN-sNF-90 measured at (i) initial state, and after water touching the mats for (ii) $1 \mathrm{~s}$ and (iii) $4 \mathrm{~s}$. (d) The MMT results of (i) PAN-sNF, (ii) PAN-sNF-15, (iii) PAN-sNF-90. 
The apparent contact angles of PS-sNF, PS-sNF-15 and PS-sNF-90 are shown in Figures 6a-6c. In contrast to that of PAN-sNF, which shows much smaller apparent water contact angle than the cast PAN film, the apparent water contact angle of PS-sNF is much larger than the cast PS film because for hydrophobic materials, a rough surface at submicron scale could greatly enlarge water contact angle owing to the lotus effect. ${ }^{24}$ In addition, the contact angle does not change with time for fairly lone, i.e. in 3 mins (Figure S5), indicating water spreading along/across the nanofibers and penetrating into the pores does not occur. However, after PS-sNF is coated with PDOPA for $15 \mathrm{~min}$, the water contact angle decreases quickly and drops to 0 in less than $4 \mathrm{~s}$, indicating that water is able to penetrate through the mat. For the smaple coated with PDOPA for 90 mins, the surface of the mats is much more hydrophilic and the water could thus move through the mat faster. This is in good aggrement with the MMT results shown in Figure $6 \mathrm{~d}$. The dashed line keeps at zero all the time for PS-sNF, indicating no water transport through the mat. PS-sNF-90 exhibits faster water transport and higher relative water content on the bottom surface than PS-sNF-15. Different from PAN-sNF-90, for PS-sNF-90, the water contents on both sides are roughly the same, which is due to the much smaller thickness of the PS mats $(\sim 20 \mu \mathrm{m})$ than the PAN mats $(\sim 80 \mu \mathrm{m})$. 


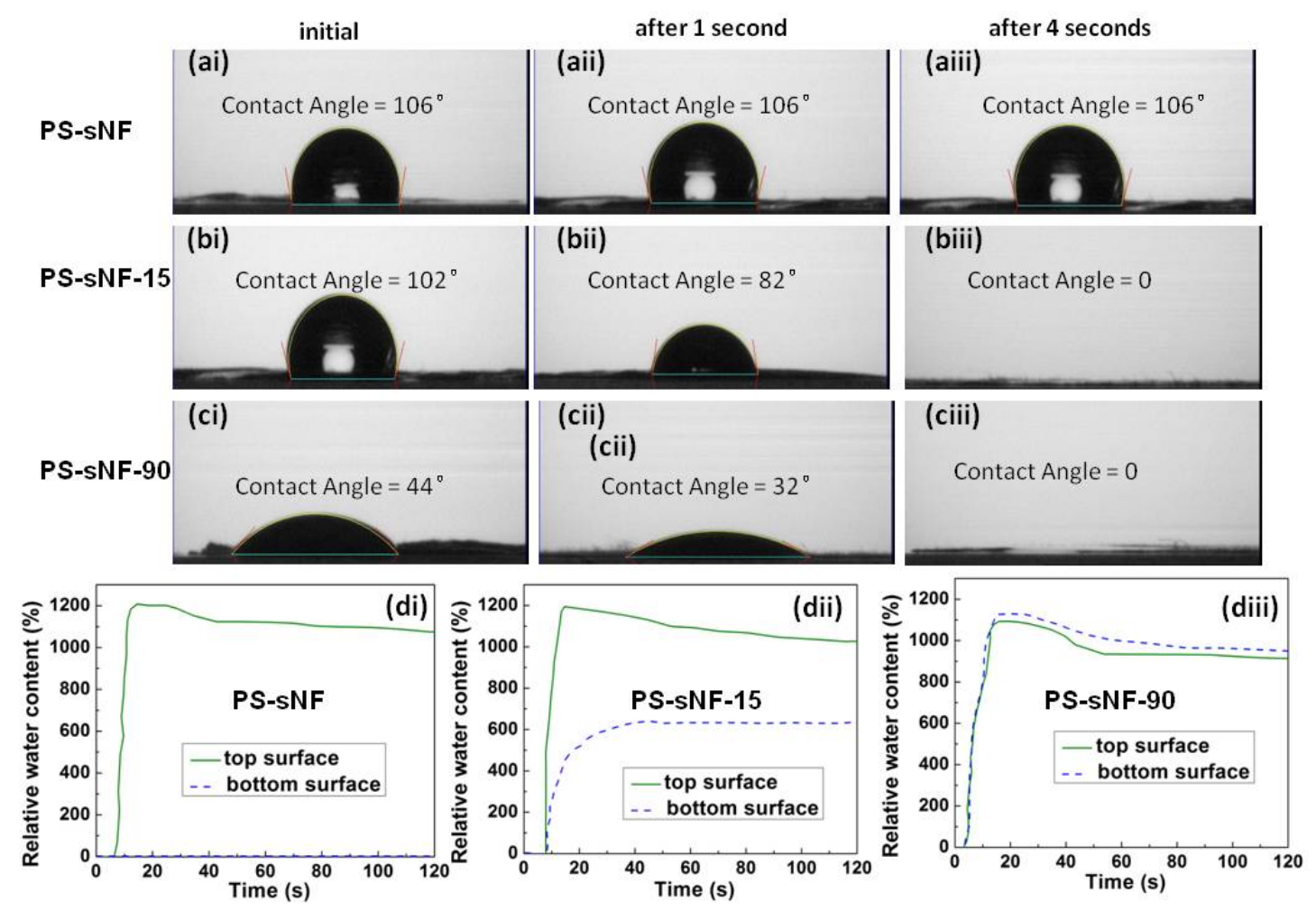

Figure 6. Apparent water contact angles of (a) PS-sNF, (b) PS-sNF-15 and (c) PS-sNF-90 measured at (i) initial state, and after water touching the mats for (ii) $1 \mathrm{~s}$ and (iii) $4 \mathrm{~s}$. (d) The MMT results of (i) PS-sNF, (ii) PS-sNF-15, (iii) PS-sNF-90.

As mentioned earlier, the nanochannels in porous PS nanofibers can in principle enhance the capillary effect. Theoretically, the narrower the channels, the stronger the capillary effect and hence the greater the capability to wick moisture. Since the size of the interpenetrating pores in the porous PS nanofibers is much smaller than the inter-fiber spaces, which are micrometers and above in size, the interpenetrating pores would probably facilitate the capillary motion more significantly. However, the apparent water contact angle of PS-pNF (Figure 7a) is only slightly smaller than that of PS-sNF (Figure 6a) and it also does not decrease with time quickly because 
these two mats have similar hydrophobic surface and submicron-scale roughness that make water spreading and penetrating difficult. When the porous PS nanofibers are coated with PDOPA for 15 min, the apparent water contact angle of PS-pNF-15 becomes significantly smaller than that of both PS-pNF and PS-sNF-15, showing enhanced capillary effect. Furthermore the contact angle of PS-pNF-15 deceases with time more quickly than that of PS-sNF-15, indicating faster water transport in PS-pNF-15. This verifies that appropriate water wettability (hydrophilicity) is a precondition for effective capillary effect. The improved water transport of PS-pNF and PSpNF-15 in comparison with that of PS-sNF and PS-sNF-15 is further confirmed by MMT results, as shown in Figure 7d. PS-pNF shows detectable water content, albeit small, on the bottom surface, indicating that even without the surface modification the capillary motion can be enhanced owing to the presence of nanochannels. The surface of PS-pNF-90 is highly hydrophilic due to much longer PDOPA coating time and hence water could be transported through the thin mat, similar to that of PS-sNF-90. PS-sNF-90 and PS-pNF-90 thus show similar MMT results. 


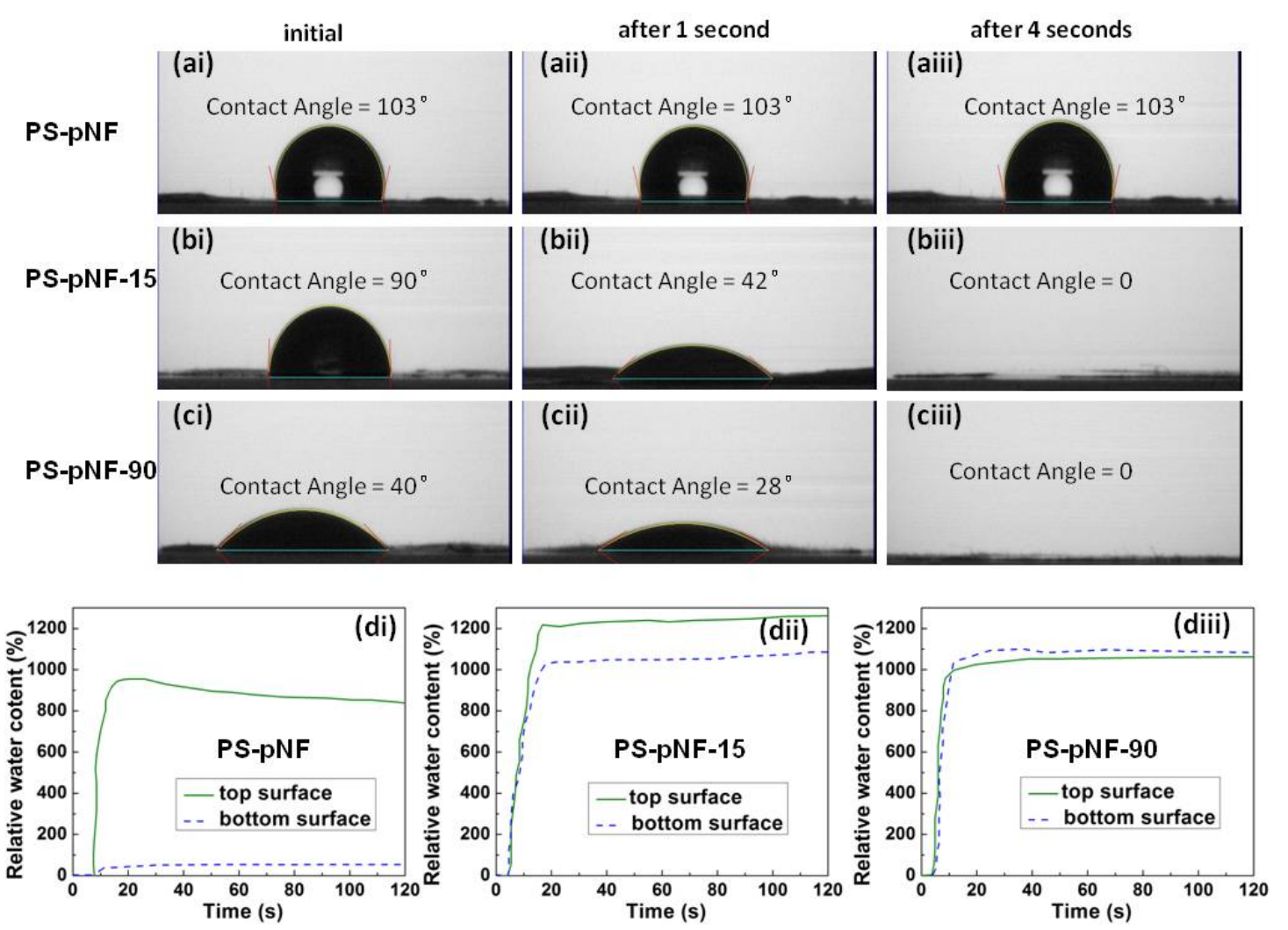

Figure 7. Apparent water contact angles of (a) PS-pNF, (b) PS-pNF-15 and (c) PS-pNF-90 measured at (i) initial state, and after water touching the mats for (ii) $1 \mathrm{~s}$ and (iii) $4 \mathrm{~s}$. (d) The MMT results of (i) PS-pNF, (ii) PS-pNF-15, (iii) PS-pNF-90.

\section{Liquid moisture transport behaviors of PAN-PS dual-layer nanofibrous mats}

As mentioned in the introduction, dual-layer textiles with hydrophobic inner layer and hydrophilic outer layer can induce push-pull effect to transport liquid moisture to the outer layer, ${ }^{4}$ while apparels with a dry inner layer would make people more comfortable. The PSpNF/PAN-sNF dual-layer nanofirous mats were fabricated by electrospinning of PS first followed by electrospinning of PAN on top of the PS nanofibers. In the cross-sectional FESEM 
image shown in Figure S6, we can see that in the interfacial area, the PS nanofibers (thicker ones) are entangled with the PAN nanofibers (thinner ones), giving rise to excellent adhesion between the two layers. This will facilitate the transport of liquid moisture from the inner to outer layer. Figure 8 shows the MMT results of the dual-layer nanofibrous mats composed of PS top layer and PAN bottom layer. It can be seen that for the mats with either PS-sNF or PS-pNF top layer without the PDOPA coating, water cannot be effectively transported to the bottom layer owing to the hydrophobic nature of PS; it has too small adhesive force with water to trigger the capillary motion and hence hinders the push-pull effect. Even though, the PS-pNF/PAN-sNF dual-layer mat still performs slightly better than its PS-sNF/PAN-sNF counterpart as evidenced by a very light increase in water content of the bottom layer of PS-pNF/PAN-sNF over time (the dash line in Figure 8(bi)). This demonstrates that the capillary motion can indeed be enhanced by creating nanopores in the nanofibers. 

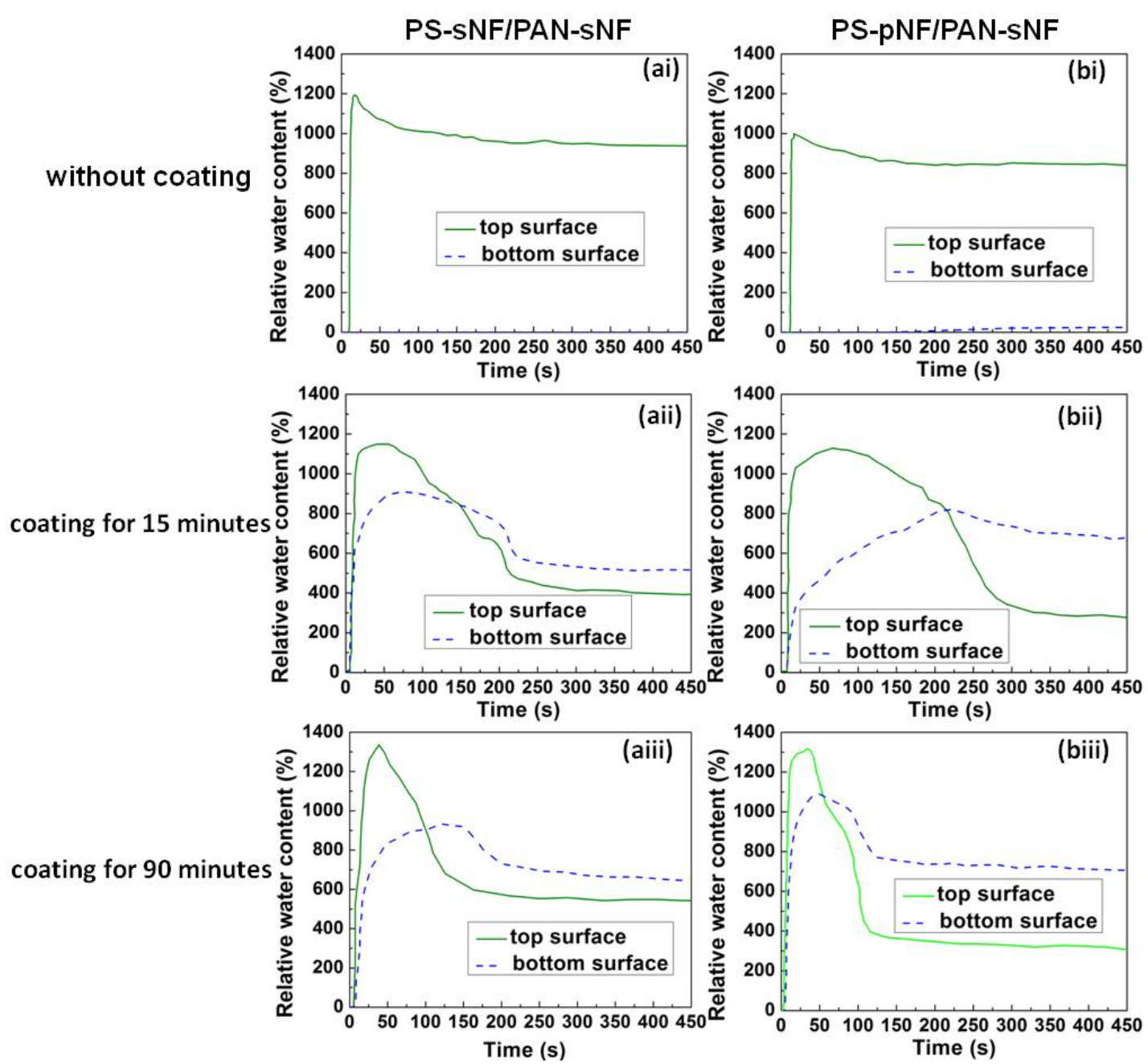

Figure 8. MMT results of the dual-layer mats with PAN-sNF as the bottom layer, and (ai, aii and aiii) PS-sNF as the top layer and (bi, bii and biii) PS-pNF as the top layer. The samples include the mats not coated with PDOPA and those coated with PDOPA for 15 and 90 mins, respectively.

For the mats coated with PDOPA, water can be effectively pushed into the pores in the top layer via caplillary motion and pulled out by the hydrophilic bottom layer. Such significant push-pull effect is clearly demonstrated in Figures 8(aii-aiii) and 8(bii-biii), where the two lines in each 
figure cross over after some time, i.e., the water content of the bottom surface becomes distinctly higher than that of the top surface, indicating that most water is successfully transported from the relatively hydrophobic top layer to the hydrophilic bottom layer. As shown earlier, compared with PS-sNF-15 and PS-sNF-90, the interpenetrating nanopores in PS-pNF-15 and PS-pNF-90 lead to slightly stronger capillary motion (slightly smaller apparent contact angles). It is striking to see that the nanopores facilitate the push-pull effect in the corresponding double-layer mats very significantly. With PS-pNF-15 and PS-pNF-90 top layers, the water content difference between the top and bottom layers of the dual-layer mats is significantly larger than that of the mats with the PS-sNF-15 and PS-sNF-90 top layers, i.e. the stronger capillary effect makes the top layer much drier. From Figures 8(bii) with 8(biii), it is clear that for the mat with the PSpNF-90 top layer the crossover of the two lines occurs within 1 min, whereas for the mat with PS-pNF-15 top layer, the crossover occurs after $3 \mathrm{~min}$, indicating that the former exhibits faster water transport speed than the latter. This can be attributed to the stronger capillary motion induced by the more hydrophilic surface in PS-pNF-90.

Figure 9 further demonstrates the effect of capillary motion on liquid moisture transport behavior of the dual-layer mats. Without the PDOPA coating, an aqueous blue ink droplet placed on the PS-pNF top layer does not spread out or penetrate much into the mat within 50 seconds (Figure 9A). Almost no ink stain is observed on the other side of the dual-layer mat. At the same time, the other ink droplet placed on the PAN bottom layer (on the folded half in Figure 9A) quickly spreads out. After 50 seconds, a very pale blue spot is observed on the backside, i.e., the PS-pNF top layer. This indicates that the droplet is mainly trapped in the PAN layer, further confirming that although water can wet the PAN layer easily, it is difficult to pass through the hydrophobic 
PS layer without the PDOPA coating. Figure 9B shows that with the PDOPA coating, the ink droplet placed on the PS-pNF-15 top layer passes through the mat quickly and spreads in both the top and the bottom layer, leaving ink spots on both sides of the mat. The color of the spot on the bottom surface is much darker, showing that the bottom surface has higher water content than the top one. It verifies that with the aid of the nanochannels, a slight improvement in hydrophilicity of the PS nanofiber surface can promote capillary motion significantly, while by controlling coating time a large hydrophilicity difference between the top and bottom layers can be retained to provide enough driving force for pulling the water out.

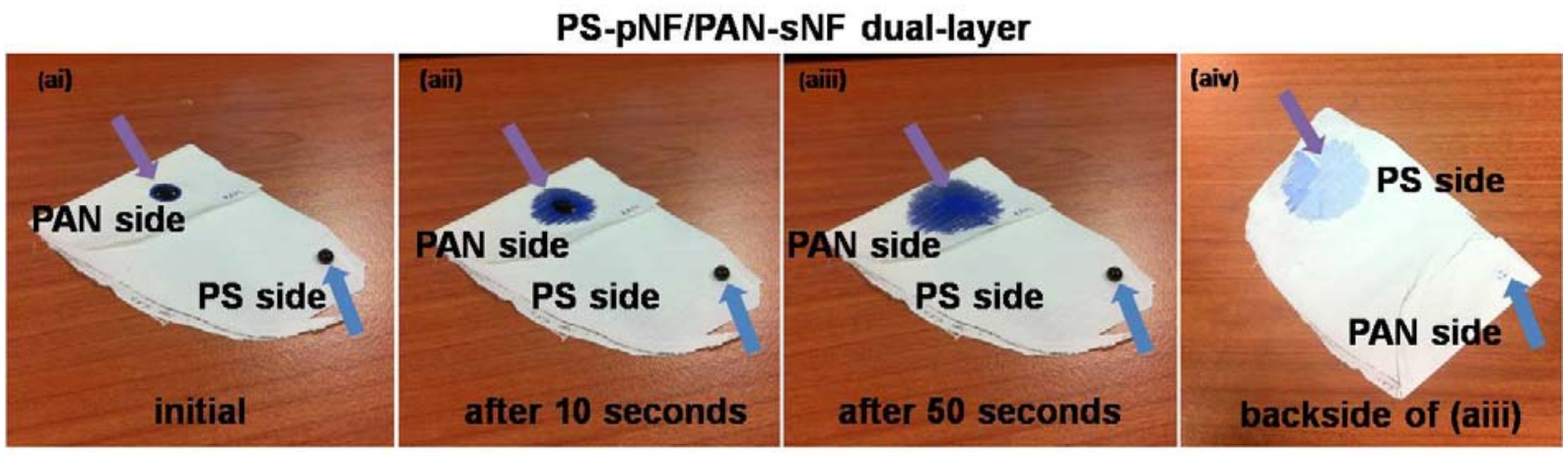

PS-pNF/PAN-sNF-15 dual-layer coated with PDOPA for 15 minutes

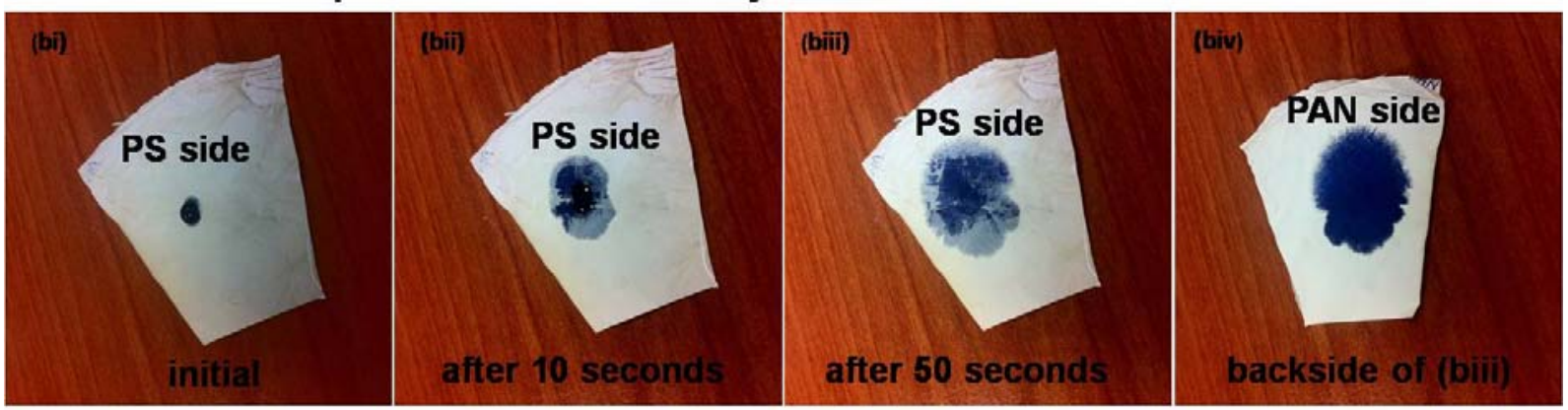

Figure 9. Pictures showing water spreading and transport abilities of (ai, aii, aiii and aiv) a PSpNF/PAN-sNF dual-layer mat without PDOPA coating and (bi, bii, biii and biv) a PS-pNF/PANsNF dual-layer mat coated with PDOPA for 15 min.

The durability of the PDOPA coating was also evaluated by comparing apparent contact angles of the PS-pNF-15/PAN-sNF dual-layer mat before and after washing. The apparent contact angle 
remains almost the same after washing, as shown in Figure S7, implying that the PDOPA coating on the nanofibers are well retained after washing.

In summary, in this study, the good moisture transport potential of electrospun nonwoven mats is validated experimentally for the first time using double-layer PAN-PS electrospun nanofibrous mats with PDOPA coatings as the testbed. It is demonstrated that by significantly reducing the size of the water transport channels through using the nanofibers with interpenetrating pores and slightly improving the hydrophilicity of the nanofiber surface, the capillary motion in the hydrophobic nanofibrous mats can be enhanced significantly. Since the nanochannels require very low hydrophilicity to trigger the capillary motion, in the dual-layer system the hydrophilicity difference between the two layers can be fairly large to give significant push-pull effect, resulting in a much drier inner layer. We believe that the approach demonstrated in this study has great potential to be extended to other base polymer systems, possibly in combination with other surface modification methods, for development of advanced sportswear and workwear. The porous electrospun nanofibers may also be used to modify conventional textiles to boost their moisture transport ability.

\section{ASSOCIATED CONTENT}

Supporting Information. FESEM micrographs of the as-spun PAN of $6 \%, 8 \%$ and $10 \%$, the MMT results of PAN of $6 \%, 8 \%$ and $10 \%$, average diameter and apparent contact angle of PAN nanofibers with different concentration, FESEM micrographs of PAN and solid PS nanofibers coated with PDA for 90 mins, FTIR spectra of the solid PS nanofibrous mat and the one coated 
with PDA for 90 mins, apparent water contact angles of PAN-sNF measured at initial state, and after water touching the mats for $1 \mathrm{~s}$ and $2 \mathrm{~s}$, apparent water contact angles of PS-sNF measured at initial state, and after water touching the mats for $1 \mathrm{~min}$ and 3 mins, a cross-sectional FESEM image of the PS-pNF-15/PAN-SNF dual-layer nanofibrous mat, apparent water contact angles of a PS-pNF/PAN-sNF dual-layer mat coated with PDOPA for 15 min before and after laundry treatment. This material is available free of charge via the Internet at http://pubs.acs.org.

\section{AUTHOR INFORMATION}

\section{Corresponding Author}

*E-mail: ASXHLu@ntu.edu.sg

\section{Author Contributions}

\#These authors contributed equally.

\section{ACKNOWLEDGEMENT}

Dong Yuliang thanks the Institute for Sport Research, Nanyang Technological University, Singapore for providing his Ph.D. scholarship to carry out this work.

\section{REFERENCES}

1. Su, C. I.; Fang, J. X.; Chen, X. H.; Wu, W. Y. Moisture Absorption and Release of Profiled Polyester and Cotton Composite Knitted Fabrics Text. Res. J. 2007, 77, 764-769. 
2. Zaman, M.; Liu, H.; Xiao, H.; Chibante, F.; Ni, Y. Hydrophilic Modification of Polyester Fabric by Applying Nanocrystalline Cellulose Containing Surface Finish Carbohydr. Polym. 2013, 91, 560-567.

3. Salas, C.; Genzer, J.; Lucia, L. A.; Hubbe, M. A.; Rojas, O. J. Water-Wettable Polypropylene Fibers by Facile Surface Treatment Based on Soy Proteins ACS Appl. Mater. Interfaces 2013, 5, 6541-6548.

4. Süpüren, G.; Oglakcioglu, N.; Ozdil, N.; Marmarali, A. Moisture Management and Thermal Absorptivity Properties of Double-Face Knitted Fabrics Text. Res. J. 2011, 0040517511402122.

5. Agarwal, S.; Greiner, A.; Wendorff, J. H. Functional Materials by Electrospinning of Polymers Prog. Polym. Sci. 2013, 38, 963-991.

6. Deitzel, J.; Kleinmeyer, J.; Harris, D. e. a.; Beck Tan, N. The Effect of Processing Variables on the Morphology of Electrospun Nanofibers and Textiles Polymer 2001, 42, 261272.

7. Huang, Z.-M.; Zhang, Y.-Z.; Kotaki, M.; Ramakrishna, S. A Review on Polymer Nanofibers by Electrospinning and Their Applications in Nanocomposites Compos. Sci. Technol. 2003, 63, 2223-2253.

8. Hussain, D.; Loyal, F.; Greiner, A.; Wendorff, J. Structure Property Correlations for Electrospun Nanofiber Nonwovens Polymer 2010, 51, 3989-3997.

9. Moon, S.; Emrick, T. High Flame Resistant and Strong Electrospun PolyacrylonitrileCarbon Nanotubes-Ochre Nanofibers Polymer 2013, 54, 1813-1819.

10. Bedford, N.; Steckl, A. Photocatalytic Self Cleaning Textile Fibers by Coaxial Electrospinning ACS Appl. Mater. Interfaces 2010, 2, 2448-2455.

11. Lee, S.; Kay Obendorf, S. Developing Protective Textile Materials as Barriers to Liquid Penetration Using Melt - Electrospinning J. Appl. Polym. Sci. 2006, 102, 3430-3437.

12. Gibson, P.; Schreuder-Gibson, H.; Rivin, D. Transport Properties of Porous Membranes Based on Electrospun Nanofibers Colloids Surf., A 2001, 187, 469-481.

13. Bagherzadeh, R.; Latifi, M.; Najar, S. S.; Gorji, M.; Kong, L. Transport Properties of Multi-Layer Fabric Based on Electrospun Nanofiber Mats as a Breathable Barrier Textile Material Text. Res. J. 2012, 82, 70-76.

14. Fashandi, H.; Karimi, M. Pore Formation in Polystyrene Fiber by Superimposing Temperature and Relative Humidity of Electrospinning Atmosphere Polymer 2012, 53, 58325849.

15. Lin, J.; Ding, B.; Yu, J. Direct Fabrication of Highly Nanoporous Polystyrene Fibers Via Electrospinning ACS Appl. Mater. Interfaces 2010, 2, 521-528.

16. Lu, P.; Xia, Y. Maneuvering the Internal Porosity and Surface Morphology of Electrospun Polystyrene Yarns by Controlling the Solvent and Relative Humidity Langmuir 2013, 29, 7070-7078.

17. Jonoobi, M.; Harun, J.; Mathew, A. P.; Hussein, M. Z. B.; Oksman, K. Preparation of Cellulose Nanofibers with Hydrophobic Surface Characteristics Cellulose 2010, 17, 299-307.

18. Martins, A.; Pinho, E. D.; Faria, S.; Pashkuleva, I.; Marques, A. P.; Reis, R. L.; Neves, N. M. Surface Modification of Electrospun Polycaprolactone Nanofiber Meshes by Plasma Treatment to Enhance Biological Performance Small 2009, 5, 1195-1206.

19. Lee, H.; Dellatore, S. M.; Miller, W. M.; Messersmith, P. B. Mussel-Inspired Surface Chemistry for Multifunctional Coatings science 2007, 318, 426-430. 
20. Yang, H.; Lan, Y.; Zhu, W.; Li, W.; Xu, D.; Cui, J.; Shen, D.; Li, G. PolydopamineCoated Nanofibrous Mats as a Versatile Platform for Producing Porous Functional Membranes $J$. Mater. Chem. 2012, 22, 16994-17001.

21. Kong, J.; Yee, W. A.; Yang, L.; Wei, Y.; Phua, S. L.; Ong, H. G.; Ang, J. M.; Li, X.; Lu, $X$. Highly Electrically Conductive Layered Carbon Derived from Polydopamine and Its Functions in Sno2-Based Lithium Ion Battery Anodes Chem. Commun. 2012, 48, 10316-10318. 22. Hu, J.; Li, Y.; Yeung, K.-W.; Wong, A. S.; Xu, W. Moisture Management Tester: A Method to Characterize Fabric Liquid Moisture Management Properties Text. Res. J. 2005, 75, 57-62.

23. Müller, M.; Keßler, B. Deposition from Dopamine Solutions at Ge Substrates: An in Situ Atr-Ftir Study Langmuir 2011, 27, 12499-12505.

24. Singh, A.; Steely, L.; Allcock, H. R. Poly [Bis (2, 2, 2-Trifluoroethoxy) Phosphazene] Superhydrophobic Nanofibers Langmuir 2005, 21, 11604-11607. 
For Table of Contents Only

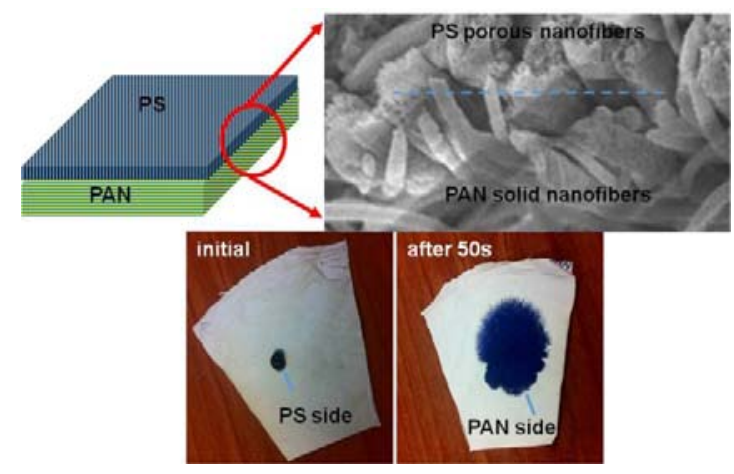

